Received: 2019-04-24

Accepted: 2019-10-02

Published: 2020-03-30

\section{Uterine Prolapse following a Transforaminal Epidural Steroid Injection: A Case Report}

\author{
Samantha Erosa, MD' \\ Houman Danesh, MD² \\ Michele Silverstein, $\mathrm{MD}^{2}$
}

\begin{abstract}
Epidural steroid injections (ESIs) for the treatment of radicular pain remains one of the most common procedures in interventional pain management. The medical literature is filled with detailed data regarding potential complications and side effects of ESIs. However, the literature, to date, has no documented cases of uterine prolapse following a transforaminal ESI. A suggested explanation for the etiology of this complication involves the proximity of the major psoas muscle to the round ligament, whereby spasm of the psoas can physically interrupt the integrity of the round ligament and result in uterine prolapse.
\end{abstract}

Key words: Transforaminal epidural steroid injection, uterine prolapse, adverse event 


\section{BACKGROUND}

The treatment of neuropathic spinal pain with an epidural injection dates back to the early 20th century when 2 French physicians, Sicard and Cathelin, caudally injected cocaine into the epidural space for the treatment of sciatica (1). In modern-day medicine, epidural steroid injection (ESI) is the most commonly performed procedure in pain management. The indication for ESI is, primarily, spinal pain or radiculopathy recalcitrant to conservative management. The procedure is performed through 1 of 3 main approaches: interlaminar, transforaminal, or caudal. Studies have shown transforaminal epidural steroid injections (TFESIs) to be superior to interlaminar and caudal ESIs, as the transforaminal approach allows for administration of the injectate directly into the ventral epidural space targeting the nerve root (2).

Procedural complications related to ESIs largely fall under 3 main categories: those associated with trauma, those associated with infection, and those secondary to drug-related systemic effects. Major procedural complications include dural puncture, epidural hematoma, spinal cord trauma, subdural injection, pneumocephalus, cauda equina syndrome, stroke, paralysis, and death. Major infectious complications include epidural abscess, discitis, and meningitis. Although major complications have been reported in the literature, the incidence is rare. In a study of 52,935 fluoroscopy-guided ESIs performed over 12 years, the incidence of ESI-related events requiring hospitalization was $0.46 \%$ and the incidence of a major complication was $0.011 \%$ (3). A separate study of 10,000 fluoroscopically directed ESIs reported zero cases of spinal cord infarct, nerve damage, or infection (4). The risk of stroke and paralysis are related to the inadvertent administration of particulate steroids intraarterially resulting in embolic infarction. However, the administration of contrast medium with fluoroscopic-guidance to ensure extraarterial needle placement in addition to the use of nonparticulate steroids for injections reduces the risk of major neurologic complications.

More commonly, minor side effects are reported by patients. These include increased back pain, pain at the injection site, numbness, paresthesias, and bruising. McGrath et al (5) published a study of 4,265 ESIs of which 3,964 were lumbar transforaminal injections and no major complications were reported. The most common complications recorded were increased pain, pain at the injection site, and persistent numbness with an incidence of $1.1 \%, 0.33 \%$, and $0.14 \%$, respectively (5). Side effects related to the administration of corticosteroids can also occur, the most common of which is facial flushing. Other side effects of corticosteroids include weight gain, hyperglycemia, osteoporosis, pituitary-adrenal axis suppression, and avascular necrosis of the bone.

\section{CASE REPORT}

The patient is a 51-year-old woman that presented with a 6-month history of low back pain with radiation into the left anterior thigh and leg. She described the pain as "lightning-like, cutting, stabbing, and like electric shocks." She denied any changes in bowel or bladder function. On physical examination, patient was diffusely tender to palpation over lumbar paraspinals. Otherwise, her examination did not reveal any sensory, motor, or reflex deficits. Slump's and straight leg raise were negative bilaterally.

Considering the provided history, symptoms, and physical examination, the patient was thought to have radicular symptoms likely due to pathology at the L3-L4 level, not resolved with conservative measures including ibuprofen and rest. Magnetic resonance imaging of the lumbar spine confirmed L3-L4 disc bulge with left foraminal disc herniation contacting the descending left $L 4$ and exiting left $L 3$ nerve root. She was scheduled for an L3 and L4 diagnostic TFESI 1 week later. Prior to the procedure, the patient's vitals were stable. She was placed in the prone position, draped, and the procedure was performed using aseptic techniques. Lidocaine $1 \%$ was administered to the skin using a 25-gauge needle. Then a 22-gauge 3.5-inch blunt tip needle was guided using fluoroscopy into the posterior superior quarter of the foramen at the left L3 and L4. Using "Roadmapping," $0.5 \mathrm{~mL}$ of Isovue 250 (Bracco Diagnostics Inc., Monroe Township, NJ) was injected via each needle, with contrast medium outlining the respective nerve root with passage into the epidural space. No intravascular contrast medium was visualized. After negative aspiration, $1 \mathrm{~mL}$ of $0.25 \%$ bupivacaine with dexamethasone $8 \mathrm{mg}$ and $4 \mathrm{~mL}$ normal saline solution were injected at each level. The needles were then flushed with normal saline solution and removed. The area was cleaned, and a sterile dressing applied. The patient tolerated the procedure well and was transferred to the postanesthesia care unit in stable condition.

The patient followed up in the office 12 days later and reported $90 \%$ to $100 \%$ pain relief that started about 24 hours after the procedure. However, the patient reported that immediately after the procedure she felt a sense of fullness in her vaginal canal. She did 
not have any associated increase in groin or thigh pain. She performed a self-examination and felt the prolapse at the opening of the introitus. She denied any pain or urinary incontinence. She denies any prior history of uterine prolapse. Physical examination at follow-up was unchanged from prior with intact sensation to light touch and 5/5 strength in bilateral lower extremities. Past medical history is remarkable for 2 vaginal births requiring forceps and an episiotomy. The patient denies any bladder, vaginal, or uterine issues prior to the epidural injection. She underwent evaluation by a gynecologist and was subsequently diagnosed with incomplete uterovaginal prolapse. Two months postinjection, at the time this manuscript was written, the uterine prolapse had not yet resolved and she was fit for a vaginal pessary.

\section{DISCUSSION}

Pelvic organ prolapse (POP) is defined as herniation of the pelvic organs to or beyond the vaginal walls. Risk factors for POP include parity, advancing age, and obesity. Among parous women, injury to the levator ani or pudendal nerve during childbirth can result in POP. Additionally, prolonged second stage of labor, high infant birth weight, and maternal age $<25$ years at first delivery are also associated with prolapse (6). When studying the effect of weight on risk for prolapse, a meta-analysis of 22 studies found overweight (body mass index [BMI] $\geq 25$ to $29.9 \mathrm{~kg} / \mathrm{m}^{2}$ ) and obese (BMI $\geq$ $30 \mathrm{~kg} / \mathrm{m}^{2}$ ) women to have a $40 \%$ and $50 \%$ increased risk of POP, respectively (7).

Many women with POP are asymptomatic. However, POP can present as a bulge or vaginal pressure. Associated symptoms include stress urinary incontinence and constipation (8). Severity of symptoms are often related to position. Symptoms are less noticeable in the morning and while laying supine. Alternatively, they can worsen as the day progresses and for women in an upright position. Treatment is indicated for symptomatic women with prolapse. Conservative treatment options include pelvic floor muscle exercises and vaginal pessaries to support the pelvic organs. Women who have failed or declined conservative management can consider surgical intervention.

The literature, to date, has no documented cases of uterine prolapse following a lumbar TFESI. The levator ani comprise a major source of support for the uterus and are innervated by the pudendal nerve. The pudendal nerve arises from the S2, S3, and S4 nerve roots. Trauma to these nerve roots could result in laxity of the pelvic floor muscles and subsequent uterine prolapse. However, trauma to the S2, S3, and S4 nerve roots in this case are unlikely considering the procedure targeted the $\mathrm{L} 3$ and $\mathrm{L} 4$ nerve roots under fluoroscopic guidance. Important to mention are the patient's increased risk factors, including BMI 24.8, history of 2 vaginal births, and advancing age. However, it is very unlikely these chronic factors that were present before the procedure would cause a sudden-onset uterine prolapse immediately postintervention. More likely, this event can be attributed to the epidural spinal injection.

A possible explanation for this adverse event involves the proximity of the psoas muscle to the round ligament. The psoas muscle is innervated by direct branches of the anterior rami of the lumbar plexus at levels L 1 to L3. It originates at the anterior, lower borders of the transverse processes of the T12-L5 vertebrae, passes over the pubis, and inserts at the lesser trochanter of the femur. The round ligaments of the uterus attach anteroinferiorly to the uterotubal junction and extend to the lateral pubis, thereby maintaining anteversion of the uterus. Therefore spinal intervention at the level of L3 and L4 nerve roots can cause spasm of the psoas muscle resulting in direct contact with the round ligament. Alternatively, the targeted nerve roots could also have resulted in a loss of muscle tension within the psoas and related fascia, with subsequent disruption of the round ligament. This direct contact, owing to either mechanism, can interrupt the structural function of the round ligament and lead to uterine prolapse in susceptible patients.

\section{CONCLUSIONS}

ESIs are a common procedure performed for pain management. Although this case presents a rare adverse event, it is important to share this significant occurrence with the medical community.

\section{Acknowledgments}

The authors wish to thank the editor of Pain Medicine Case Reports for their guidance and assistance with this manuscript. 


\section{REFERENCES}

1. Sicard A. Les injections medicamenteuses extradurales par voie sacro-coccygienne. CR Soc Biol 1901; 53:396-398.

2. Rho ME, Tang C-T. The efficacy of lumbar epidural steroid injections: Transforaminal, interlaminar, and caudal approaches. Phys Med Rehabil Clin N Am 2011; 22:139-148.

3. Lee JW, Lee E, Lee GY, Kang Y, Ahn JM, Kang HS. Epidural steroid injection-related events requiring hospitalisation or emergency room visits among 52,935 procedures performed at a single centre. Eur Radiol 2017; 28:418-427.

4. Manchikanti L, Malla Y, Wargo BW, Cash KA, Pampati V, Fellows B. A prospective evaluation of complications of 10,000 fluoroscopically directed epidural injections. Pain Physician 2012; 15:131140.
5. Mcgrath JM, Schaefer MP, Malkamaki DM. Incidence and characteristics of complications from epidural steroid injections. Pain Med 2011; 12:726-731.

6. Moalli PA, Jones Ivy S, Meyn LA, Zyczynski HM. Risk factors associated with pelvic floor disorders in women undergoing surgical repair. Obstet Gynecol 2003; 101:869-874.

7. Giri A, Hartmann KE, Hellwege JN, Edwards DRV, Edwards TL. Obesity and pelvic organ prolapse: A systematic review and meta-analysis of observational studies. Am J Obstet Gynecol 2017; 217:11-26.e3

8. Iglesia $C B$, Smithling KR. Pelvic organ prolapse. Am Fam Physician 2017; 96:179-185. 